\title{
Novel monoclonal antibodies against pancreatic juice from pancreatic cancer patients and their possible application in differential diagnosis
}

\author{
MASATO HAMABASHIRI $^{1}$, AYA DAICHOU ${ }^{1}$, MAKIKO YASUMOTO $^{2}$, \\ SACHIKO OGASAWARA $^{2}$, TAKUYA NISHINAKAGAWA ${ }^{1}$, MUNECHIKA ENJOJI ${ }^{1}$, \\ KOUICHI OHSHIMA ${ }^{2}$, HIROHISA YANO ${ }^{2}$ and MANABU NAKASHIMA ${ }^{1}$ \\ ${ }^{1}$ Department of Immunological and Molecular Pharmacology, Faculty of Pharmaceutical Science, \\ Fukuoka University, Jonan-ku, Fukuoka 814-0180; ${ }^{2}$ Department of Pathology, \\ Kurume University School of Medicine, Kurume, Fukuoka 830-0011, Japan
}

Received March 29, 2011; Accepted May 6, 2011

DOI: $10.3892 /$ ijmm.2011.717

\begin{abstract}
Pancreatic cancer (PC) has a poor clinical prognosis with a $<10 \% 5$-year survival rate. Because there are no specific biomarkers of PC, it is difficult to detect small PC tumors and most patients are diagnosed at an advanced stage. Specific biomarkers are useful tools for the early detection of cancer. However, PC-related biomarkers, such as CA19-9 lack specificity and sensitivity. In this study, we took an immunological approach to establish novel monoclonal antibodies (mAbs) specific for the pancreatic juice from PC patients, which would be potentially useful in the diagnosis of PC. Mice were immunized by subtractive immunization using mixed pancreatic juices from chronic pancreatitis and PC patients as the tolerogen and the immunogen, respectively. After screening by Western blotting, four mAbs were obtained: 2P-1-2-1, 2P-1-17-1, 6P-32-4 and 7P-9-11-6. The mAb 2P-1-2-1 showed reactivity against the tolerogen at 115 and $120 \mathrm{kDa}$, but only the $120-\mathrm{kDa}$ antigen was also reactive to the immunogen. The mAb 2P-117-1 showed an intense smear reactivity at $\sim 150 \mathrm{kDa}$ against the immunogen. Finally, the mAbs 6P-3-2-4 and 7P-9-11-6 showed PC-specific reactivity to the immunogen at $>250 \mathrm{kDa}$ and at $\sim 70 \mathrm{kDa}$, respectively. We propose that investigation of pancreatic juice samples with these mAbs may enable us to perform reliable differential diagnosis of benign and malignant diseases. Furthermore, we demonstrated that subtractive
\end{abstract}

Correspondence to: Dr Manabu Nakashima, Department of Immunological and Molecular Pharmacology, Faculty of Pharmaceutical Science, Fukuoka University, 8-19-1 Nanakuma, Jonan-ku, Fukuoka 814-0180, Japan

E-mail: nakashim@adm.fukuoka-u.ac.jp

Key words: pancreatic cancer, biomarker, subtractive immunization, monoclonal antibody immunization is a useful method for producing mAbs specific for the pancreatic juice from PC patients.

\section{Introduction}

Pancreatic cancer (PC) is the fifth leading cause of cancer death and the number of deaths from PC is increasing. PC has a poor clinical prognosis and its 5-year survival rate is $<10 \%(1,2)$. Despite recent improvements in imaging and endoscopic approaches, most patients are diagnosed at an advanced stage. Improving the prognosis of PC is critically dependent on earlier detection (3). Specific biomarkers are useful tools for the early detection of cancer. However, PC-related biomarkers such as CA19-9 lack specificity and sensitivity. Thus, these biomarkers are not sufficient to detect early-stage PC, although they are useful to determine prognosis and predict recurrence after surgical tumor resection (4-7). A specific biomarker for PC is needed.

Many studies have reported that pancreatic juice is a useful specimen to identify PC-related antigens, because it is secreted from the pancreatic ductal lumen from where PC cells arise $(5,8,9)$. Particular antigens in pancreatic juice with specific and/or high expression in PC may be useful to differentiate between benign and malignant disease. Here, we used an immunological approach to establish monoclonal antibodies specific for pancreatic juice from PC patients.

Because pancreatic juice from PC patients contains many antigens that are irrelevant to the cancer, it might be difficult to induce antibodies against PC-specific antigens. Thus, we used a subtractive immunization method. Subtractive immunization is an efficient way to produce antibodies against poorly immunogenic or rare antigens (10-12). Mice were tolerized against the tolerogen using the immunosuppressive drug cyclophosphamide (Cy). Cy preferentially eliminates $\mathrm{T}$ and $\mathrm{B}$ cells activated by the tolerogen $(11,13)$. After tolerization, mice were immunized with the immunogen. Because the tolerogen-related $\mathrm{T}$ and $\mathrm{B}$ cells have been eliminated by $\mathrm{Cy}$, this is expected to induce a specific and/or intense immune reactivity to the immunogen-specific antigens. In this study, we immunized mice with pancreatic juice from PC patients 
after tolerizing the mice against the pancreatic juice from chronic pancreatitis patients, to establish PC-specific monoclonal antibodies (mAbs).

\section{Materials and methods}

Antigen preparation. All pancreatic juices were the remainders of those which were collected for the clinical diagnosis and used in this study with permission of The Ethics Committe of Kurume University. Mixed pancreatic juices from patients with chronic pancreatitis and PC were used as the tolerogen and immunogen, respectively. The protein concentration in each composite was measured by the BCA assay (Thermo Fisher Scientific, Rockford, IL).

Reagents. Horseradish peroxidase-conjugated anti-CA19-9 monoclonal antibody (CanAg CA19-9 EIA) was purchased from Fujirebio Diagnostic AB (Gothenburg, Sweden). The anti-CEA antibody (1C11) was purchased from HyTest Ltd. (Turku, Finland). Peroxidase-conjugated goat anti-mouse $\operatorname{IgG}(\mathrm{H}+\mathrm{L})$, $\operatorname{IgG}(\gamma$-chain specific) and $\operatorname{IgM}(\mu$-chain specific) were purchased from Jackson ImmunoResearch (Baltimore, MD). The o-sialoglycoprotein endopeptidase was purchased from Cedarlane Laboratories (Ontario, Canada). Neuraminidase, $\mathrm{N}$-glycosidase and O-glycosidase were purchased from Roche Applied Science (Penzberg, Germany).

Subtractive immunization. The immunization protocol was performed as previously described (10). Briefly, BALB/c female mice, 5-6 weeks old, were obtained from Kyudo (Saga, Japan). On Day 0, sera were collected. On Day 1, $25 \mu \mathrm{g}$ of tolerogen (mixed inflammatory pancreatic juice) diluted with PBS were mixed with ImmunEasy Mouse Adjuvant (Qiagen $\mathrm{GmbH}$, Hamburg, Germany) and injected s.c. into the mice. Twenty-four and $48 \mathrm{~h}$ after the first injection of tolerogen, $200 \mathrm{mg} / \mathrm{kg}$ Cy (Sigma-Aldrich, Tokyo, Japan) was injected i.p. On Day 14, $25 \mu \mathrm{g}$ of tolerogen in PBS was injected i.p. without adjuvant followed by injection of Cy 24 and $48 \mathrm{~h}$ later. We performed this cycle every 2 weeks, twice more. Three days after the last injection of tolerogen, sera were collected. On Day 52,25 $\mu \mathrm{g}$ of immunogen (mixed cancerous pancreatic juice) diluted with PBS was mixed with ImmunEasy Mouse Adjuvant and injected s.c. into tolerized mice. On Days 59 and 66, the immunogen without adjuvant was injected i.p. Three days after the last injection of immunogen, sera were collected and investigated for reactivity to the tolerogen and immunogen. All procedures regarding animal care and use were carried out according to regulations established by the Experimental Animal Care and Use Committee of Fukuoka University.

Establishment of mAb-producing hybridoma cells. Immunized mice were boosted with immunogen. Three days after the boost, splenocytes were harvested and fused with X63.Ag8.653 mouse myeloma cells using polyethylene glycol 1500 (Roche) according to the manufacturer's protocol and cultured in HAT medium (Sigma-Aldrich). For screening the antibody-producing hybridoma cells, the reactivities of the cell culture media against the tolerogen and immunogen were investigated by Western blotting using a screener blotter apparatus (Sanplatec, Osaka, Japan). Hybridoma cells that showed specificity for the

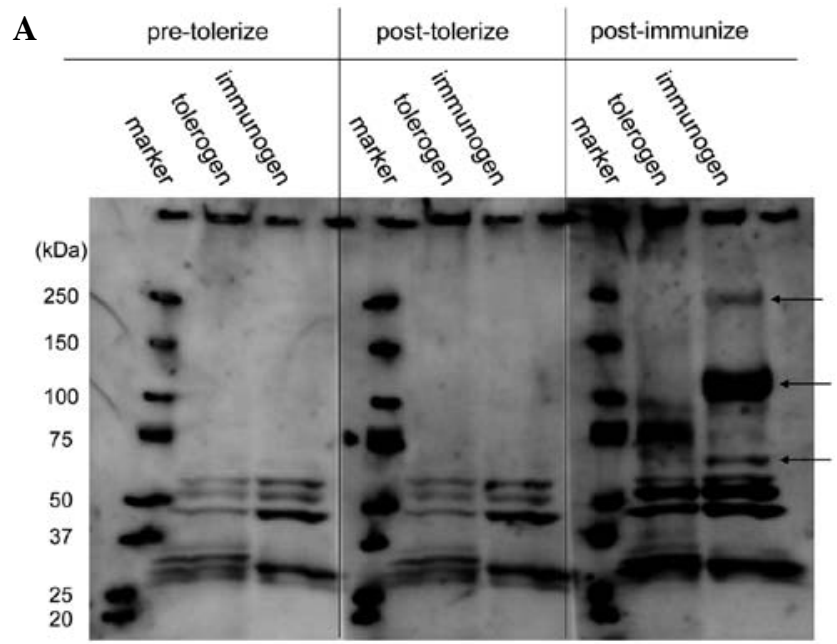

B

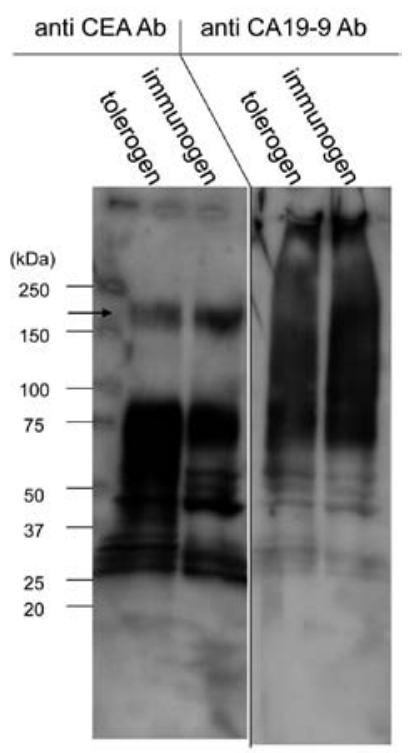

Figure 1. Reactivity of the antisera against the tolerogen and immunogen. (A) Post-immunized serum showed specific reactivity against the immunogen. (B) The characteristics of its reactivity were different from those of the anti-CEA Ab (1C11) and the anti-CA19-9 Ab.

tolerogen or immunogen were subcloned by limiting dilution to establish the mAbs.

Screener blotter analysis. The tolerogen and immunogen were electrophoresed on SDS-PAGE gels and transferred onto a PVDF membrane. The membrane was incubated with Blocking One (Nacalai Tesque, Inc., Kyoto, Japan). After washing with TBST, the membrane was placed in a screener blotter apparatus. Hybridoma supernatants were applied to each lane and incubated at room temperature for $1 \mathrm{~h}$ with shaking. After washing in TBST, the membrane was incubated with peroxidase-conjugated goat anti-mouse IgG $(\mathrm{H}+\mathrm{L})$. After washing, the reacted bands were visualized using the ECL Advance Western Blotting Detection kit (GE Healthcare BioSciences, Tokyo, Japan) and analyzed with a FluorChem image analyzer (Alpha Innotech, CA).

Western blotting. Pancreatic juices were dissolved in SDS sample buffer with reducing reagent. Proteins were electro- 


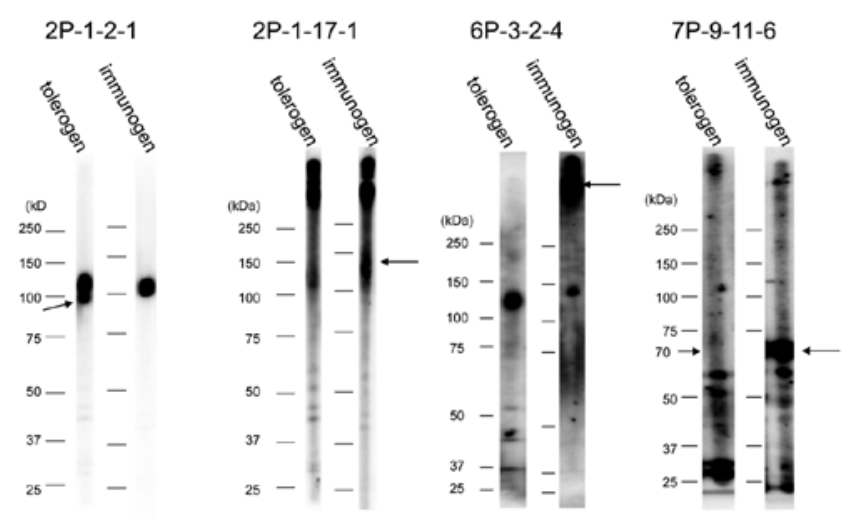

Figure 2. Screening of antibody-producing hybridoma cells. Representative data from the 13 immunogen-specific mAbs and the 4 tolerogen-specific mAbs identified by screening hybridoma cell cultured media using a screener blotter apparatus.

phoresed on SDS-PAGE gels and transferred onto PVDF membranes. The membranes were incubated with Blocking One, followed by incubation with primary antibodies. After washing, the membrane was incubated with peroxidaseconjugated goat anti-mouse $\operatorname{IgG}(\mathrm{H}+\mathrm{L}), \operatorname{IgG}(\gamma$-chain-specific) or $\operatorname{IgM}$ ( $\mu$-chain-specific) secondary antibody. Immunoreactive proteins were visualized using an ECL Advance Western Blotting Detection kit and analyzed with a FluorChem image analyzer.

Enzymatic treatment of pancreatic juice. Each sample of pancreatic juice was treated with neuraminidase $(10 \mathrm{mU} / \mathrm{ml})$, neuraminidase $(10 \mathrm{mU} / \mathrm{ml})+\mathrm{N}$-glycosidase $(20 \mathrm{U} / \mathrm{ml})$, or neuraminidase $(10 \mathrm{mU} / \mathrm{ml})+\mathrm{N}$-glycosidase $(20 \mathrm{U} / \mathrm{ml})+$ O-glycosidase $(10 \mathrm{mU} / \mathrm{ml})$ at $37^{\circ} \mathrm{C}$ for $24 \mathrm{~h}$. Treatment with o-sialoglycoprotein endopeptidase was performed at $37^{\circ} \mathrm{C} 4 \mathrm{~h}$. Each treated pancreatic juice sample was analyzed by Western blotting.

\section{Results}

Subtractive immunization. We investigated the reactivity of antisera against the tolerogen and immunogen by Western blotting. Post-immune serum showed specific reactivity for the immunogen, although there was no remarkable reactivity in post-tolerized serum (Fig. 1A). In addition, the reactive character of the post-immune serum was different from that of antiCA19-9 Ab and anti-CEA Ab (1C11), despite the fact that these antigens were detected in the tolerogen and the immunogen (Fig. 1B).

Screening of hybridoma cells. Splenocytes showing specific reactivity to the immunogen were fused with X63.Ag8.653 mouse myeloma cells. For screening the antibody-producing hybridoma cells, we investigated the reactivity of the culture media against the tolerogen and immunogen using a screener blotter. This apparatus has multiple lanes with access to the protein-bound membrane, enabling simultaneous screening of many hybridoma cell lines. We compared the reactivity of the hybridoma cell culture media against the tolerogen and the immunogen. We obtained 13 immunogen-specific and 4
A

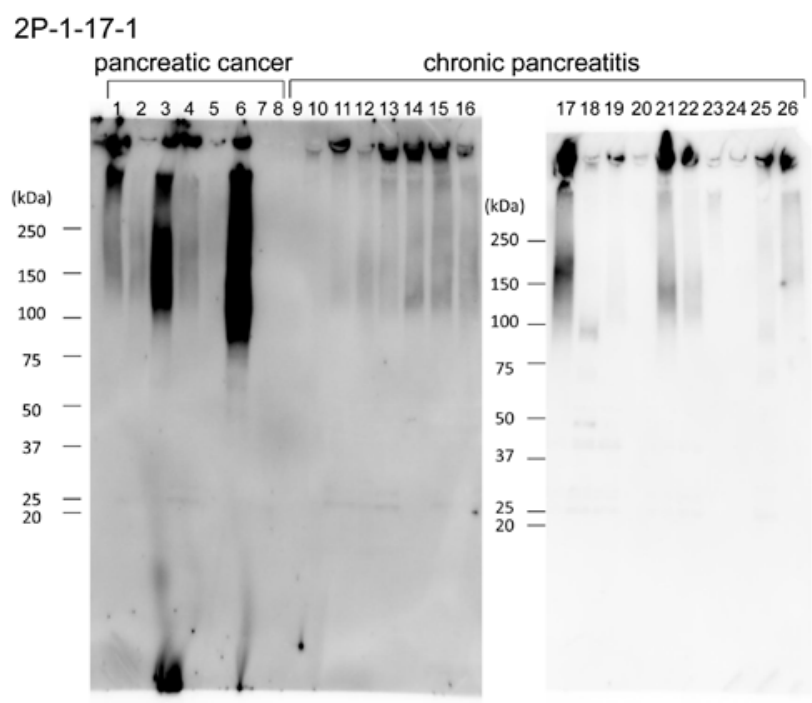

B

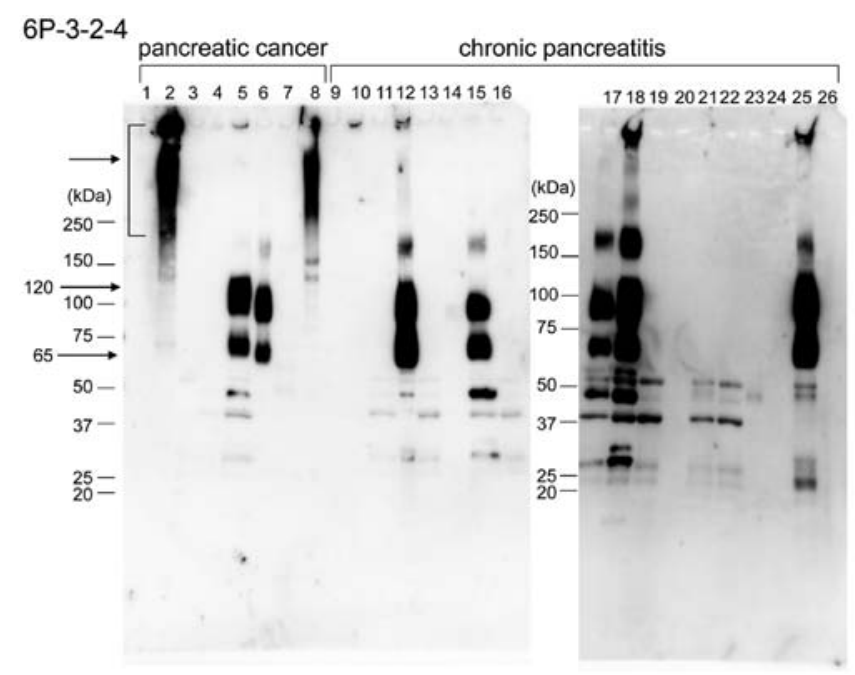

C

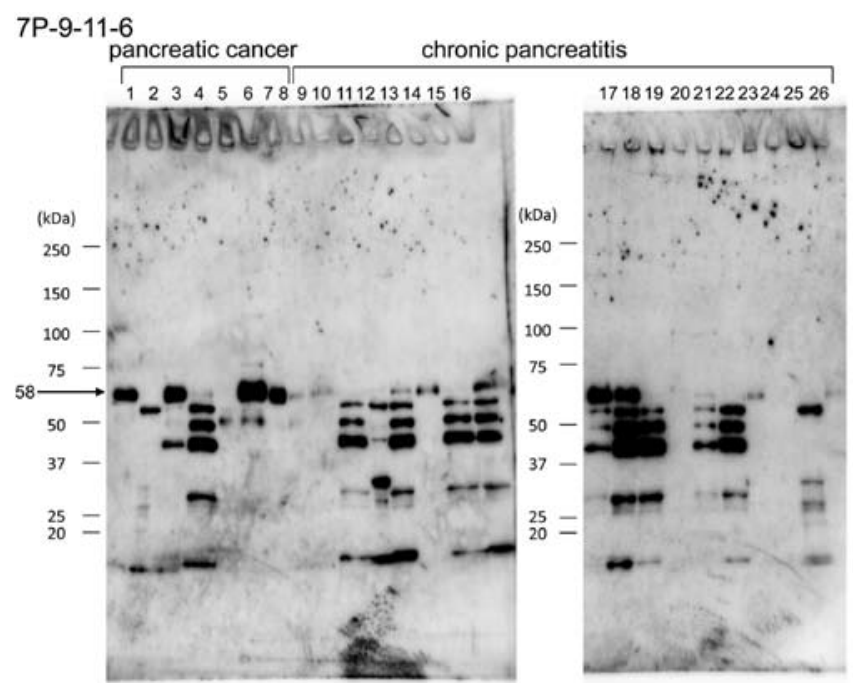

Figure 3. Analysis of the $\mathrm{mAb}$ reactivities against pancreatic juice sample by Western blotting. Three of the established mAbs showed specific or intense reactivity against pancreatic juice from PC patients: (A) 2P-1-17-1 showed an intense smear reactivity, (B) 6P-3-2-4 showed a specific reactivity at $>250 \mathrm{kDa}$, (C) 7P-9-11-6 showed an intense band at $58 \mathrm{kDa}$. Lanes 1-8, pancreatic juice from PC patients; lanes 9-26, pancreatic juice from chronic pancreatitis patients. 
A

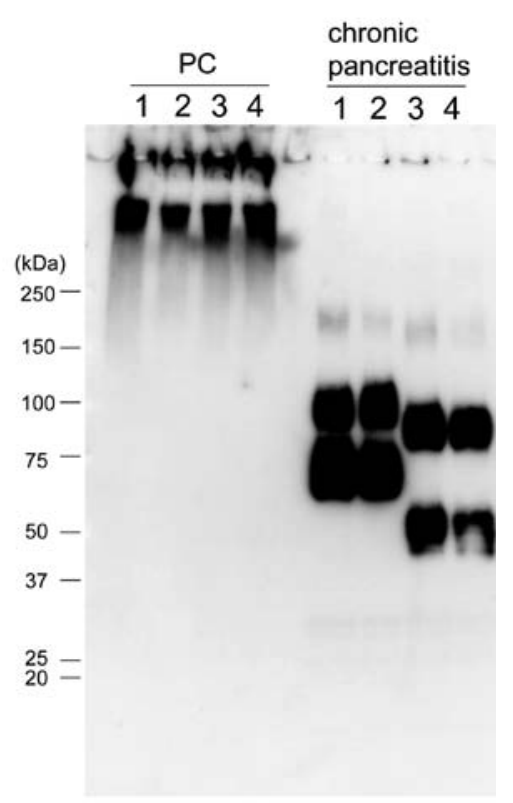

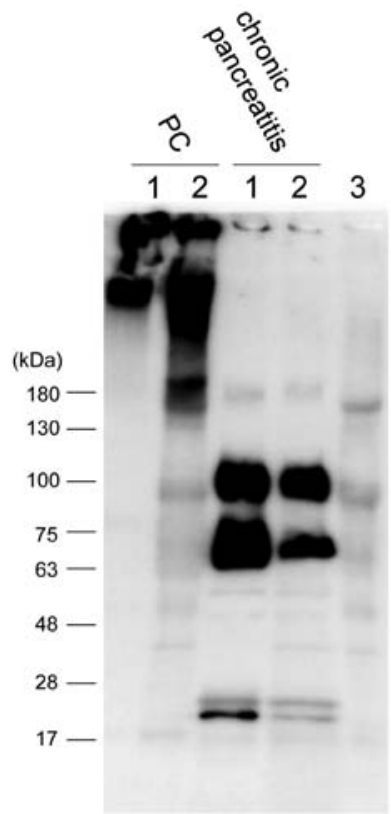

Figure 4. Enzymatic treatment of pancreatic juice samples. 6P-3-2-4-positive pancreatic juices were treated with each enzyme. (A) Pancreatic juice samples from chronic pancreatitis patients were deglycosylated by treatment with neuraminidase and $\mathrm{N}$-glycosidase. Lane 1, untreated; 2 , neuraminidase (10 mU/ml); 3, neuraminidase $(10 \mathrm{mU} / \mathrm{ml})+\mathrm{N}$-glycosidase $(20 \mathrm{U} / \mathrm{ml}) ; 4$, neuraminidase $(10 \mathrm{mU} / \mathrm{ml}),+\mathrm{N}$-glycosidase $(20 \mathrm{U} / \mathrm{ml})+\mathrm{O}$-glycosidase $(10 \mathrm{mU} / \mathrm{ml})$. (B) Pancreatic juice samples from PC patients were degraded by o-sialoglycoprotein endopeptidase. Lane 1, untreated; 2 , enzyme-treated pancreatic juice; 3 , enzyme only.

tolerogen-specific mAbs. For instance, 2P-1-2-1 showed reactivity against the tolerogen at 115 and $120 \mathrm{kDa}$, but only the $120-\mathrm{kDa}$ band was reactive against the immunogen. $2 \mathrm{P}-1-17-1$ showed intensive smear reactivity against the immunogen at $\sim 150 \mathrm{kDa}$. 6P-3-2-4 and 7P-9-11-6 showed specific reactivity against the immunogen at $>250 \mathrm{kDa}$ and $\sim 70 \mathrm{kDa}$, respectively. (Fig. 2).

Western blotting of pancreatic juice samples from PC and chronic pancreatitis patients. To investigate the reactivity and specificity of these mAbs, we performed Western blotting using pancreatic juice samples from 8 patients with $\mathrm{PC}$ and 18 patients with chronic pancreatitis. mAb 2P-1-17-1 showed strong reactivity against the pancreatic juices from $\mathrm{PC}$ patients (Fig. 3A). mAb 6P-3-2-4 showed specific reactivity at $>250 \mathrm{kDa}$ against only some PC-derived pancreatic juice samples and other reactive bands $(65-120 \mathrm{kDa})$ common to $\mathrm{PC}$ and chronic pancreatitis patients (Fig. 3B). mAb 7P-9-11-6 reacted with a $58-\mathrm{kDa}$ antigen in the pancreatic juice samples from PC patients (Fig. 3C). The other mAbs showed similar reactivity against the $\mathrm{PC}$ and pancreatitis pancreatic juices, and thus were not cancer-specific.

Enzymatic treatment of pancreatic juice. We performed enzymatic treatment of the pancreatic juice samples with neuraminidase, $\mathrm{N}$-glycosidase and $\mathrm{O}$-glycosidase to examine the alteration in reactivity of the mAbs. We detected an increased in the lower molecular weight bands among the 6P-3-2-4 antigens in the pancreatic juice from chronic pancreatitis patients after treatment with neuraminidase and $\mathrm{N}$-glycosidase (Fig. 4A). On the other hand, 6P-3-2-4 antigens $>250 \mathrm{kDa}$, which were detected only in the pancreatic juice from PC patients, were digested by treatment with o-sialoglycoprotein endopeptidase (Fig. 4B).

\section{Discussion}

Many studies have reported that pancreatic juice is a rich source of proteins released directly from PC cells, and is an available and useful specimen to identify PC-related antigens $(5,8,9)$. To produce monoclonal antibodies specific for pancreatic juice from PC patients, we immunized mice using subtractive immunization. This method is useful to produce antibodies specific to the antigen that are poorly immunogenic or have low abundance of the immunogen (10-12). Indeed, in this study, post-immunized mouse serum showed immunogenspecific bands that were different from CA19-9 and CEA, despite an abundance of these antigens in both the tolerogen and immunogen. By screening hybridoma cell lines, we obtained 13 immunogen-specific mAbs and 4 tolerogen-specific mAbs. This indicates that subtractive immunization is suitable for the generation of antibodies specific for cancerous pancreatic juice. Furthermore, using Western blotting we could evaluate the reactivity of the mAbs, including identifying the molecular weight of the antigens.

To select mAbs specific for pancreatic juice from PC patients, we investigated the reactivity of these mAbs against pancreatic juice samples from $\mathrm{PC}$ and chronic pancreatitis patients by Western blotting. Three mAbs (2P-1-17-1, 6P-3-2-4 and 7P-9-11-6) were obtained that showed specific reactivity for pancreatic juice from $\mathrm{PC}$ patients. 6P-3-2-4 showed reactivity bands $>250 \mathrm{kDa}$ and $65-120 \mathrm{kDa}$; the band at $>250 \mathrm{kDa}$ was specific for PC. In addition, each band was sensitive to treatment with different enzymes related to the carbohydrate chain. The band at $>250 \mathrm{kDa}$ was degraded by o-sialoglycoprotein endopeptidase, whereas the bands at 65-120 kDa were deglycosylated by neuraminidase and $\mathrm{N}$-glycosidase, indicating that these 6P-3-2-4 antigens have different carbohydrate chains. Many studies have reported aberrant glycosylation of 
cell surface proteins in oncogenically transformed cells (14-17). Thus, the $>250 \mathrm{kDa}$ PC-specific antigen in 6P-3-2-4 might be a cancer-related antigen with aberrant glycosylation; further investigation is required to confirm this. In addition, 2P-1-17-1 and 7P-9-11-6 showed intense reactivity in PC-derived pancreatic juice at $\sim 150$ and $58 \mathrm{kDa}$, respectively. Because 6P-3-2-4 showed reactivity bands common to $\mathrm{PC}$ and chronic pancreatitis patients by Western blotting, we believed that we would not be able to detect $\mathrm{PC}$-specific reactivity for this mAb by ELISA or immunohistochemistry. Indeed, our preliminary examinations using these other methods showed no PC-specific reactivity (data not shown). Because Western blotting also reveals the molecular weight of the antigens, this method may be reliable for differential diagnosis of benign and malignant disease. We are currently identifying the antigens recognized by these mAbs and examining their cancer specificity.

\section{Acknowledgements}

This study was supported by the Japan Society for the Promotion of Science (JPSP) KAKENHI (Grant-in-Aid Challenging Exploratory Research: 21659151).

\section{References}

1. Matsuno S, Egawa S, Fukuyama S, et al: Pancreatic cancer registry of the Japan Pancreas Society. Summary of 20 years. Pancreas 28: 219-230, 2004.

2. Seino T, Nakadaira $\mathrm{H}$, Endoh $\mathrm{K}$ and Yamamoto $\mathrm{M}$ : Changes in pancreatic cancer mortality, period patterns, and birth cohort patterns in Japan: analysis of mortality data in the period 1968-2002. Environ Health Prev Med 13: 234-242, 2008.

3. Egawa S, Takeda K, Akada M, Abe H, Yokoyana T, Motoi F, Fukuyama S, Sunamura M and Matsuno S: Small pancreatic cancer: from the national pancreatic cancer registry. J Jpn Pancreas Soc 19: 558-565, 2004 (In Japanese).
4. Koopmann J, Fedarko NS, Jain A, et al: Evaluation of osteopontin as biomarker for pancreatic adenocarcinoma. Cancer Epidemiol Biomarkers Prev 13: 487-491, 2004.

5. Chen R, Pan S, Brentnall TA and Aebersold R: Proteomic profiling of pancreatic cancer for biomarker discovery. Mol Cell Proteomic 4: 523-533, 2005.

6. Steinbrg W: The clinical utility of the CA19-9 tumor-associated antigen. Am J Gastroenterol 85: 350-355, 1990.

7. Takayama R, Nakagawa H, Saai A, et al: Serum tumor antigen REG4 as a diagnostic biomarker in pancreatic ductal adenocarcinoma. J Gastroenterol 45: 52-59, 2010.

8. Grote $\mathrm{T}$ and Logsdon CD: Progress on molecular markers of pancreatic cancer. Curr Opin Gastroenterol 23: 508-514, 2007.

9. Tonack S, Aspinall-O'Dea M, Neoptolemos JP and Costello E: Pancreatic cancer: proteomic approaches to a challenging disease. Pancreatology 9: 567-576, 2009.

10. Sleister HM and Rao AG: Subtractive immunization: a tool for the generation of discriminatory antibodies to proteins of similar sequence. J Immunol Methods 26: 213-220, 2002.

11. Zijlstra A, Testa JE and Quigley JP: Targeting the proteome/ epitome, implementation of subtractive immunization. Biochem Biophys Res Commun 202: 733-744, 2003.

12. Sleister HM and Rao AG: Strategies to generate antibodies capable of distinguishing between proteins with $>90 \%$ amino acid identity. J Immunol Methods 252: 121-129, 2001.

13. Matthew WD and Sandrock AW Jr: Cyclophosphamide treatment used to manipulate the immune response for the production of monoclonal antibodies. J Immunol Methods 100: 73-82, 1987.

14. Dennis J, Waller C, Timpl R and Schirrmacher V: Surface sialic acid reduces attachment of metastatic tumor cells to collagen type IV and fibronectin. Nature 300: 274-276, 1982.

15. Uemura T, Shiozaki K, Yamaguchi K, et al: Contribution of sialidase NEU1 to suppression of metastasis of human colon cancer cells through desialylation of integrin beta4. Oncogene 28: 1218-1229, 2009.

16. Dube DH and Bertozzi CR: Glycans in cancer and inflammation - potential for therapeutics and diagnostics. Nat Rev Drug Discov 4: 477-488, 2005

17. Hakomori S: Glycosylation defining cancer malignancy: new wine in an old bottle. Proc Natl Acad Sci USA 99: 10231-10233, 2002. 